\title{
Inmigración a Israel en la actualidad: la Aliyá y su significación contemporánea
}

Immigration to Israel today: the Aliyah and its contemporary significance

\author{
Claudia Stern W \\ pulga93@hotmail.com \\ Universidad de Tel Aviv \\ Israel
}

\section{Resumen}

El presente artículo está basado en la investigación presentada como tesis de máster en la Escuela de Estudios sobre la Cultura Shirley y Leslie Porter, Unidad para la Investigación de la Cultura de la Universidad de Tel Aviv en mayo de 2008. La investigación radicó básicamente en indagar la forma en cómo se desarrolló el proceso de inmigración a Israel de un joven inmigrante en estos días y como éste percibe su nueva realidad. Esta investigación está basada en los testimonios de nueve jóvenes entre 20 y 35 años de edad, provenientes de Argentina y Chile que inmigraron a Israel en el transcurso del año 2005. Los datos fueron recolectados a través de entrevistas en profundidad desarrolladas en el año 2007. La investigación indagó en los entornos pre inmigración, percepciones y expectativas previas, las razones que motivaron la decisión de inmigrar a Israel y de igual forma también se analiza el proceso post inmigración, el desarrollo de los investigados en este nuevo escenario cultural, más globalizado y más participativo, cómo lo perciben y cómo se ven a ellos mismos una vez en Israel. A partir de lo cual se desprenden conclusiones e hipótesis.

Palabras clave: Inmigración - Identidad - Cultura - Aculturación

\begin{abstract}
This article is based on a master-thesis investigation presented in june 2008 to The Shirley and Leslie Porter School for Cultural Studies at the Tel Aviv University. The main focus of this investigation was the development of young people throughout their process of immigration to Israel, and how they perceive their new reality. The core of this research is the testimony of nine young-adults, between the ages of 20 and 35, who immigrated to Israel from Argentina and Chile throughout the year 2005. The data was collected by means of in-depth interviews which took place in the year 2007 . The research has a two-fold
\end{abstract}


approach for analysis. The first revolves around pre-migratory surroundings, perceptions and expectations, as well as the reasons and motivations which prompted the decision to immigrate to Israel. The second pan of the analysis revolves around the post-migratory process, the development of the objects in this new culture, more global and more engaging environment, how they perceive it, and how they see themselves once in Israel. Conclusions and hypotheses follow this analysis.

Keywords: Migration - Emigration - Identity - Culture - Acculturation

\section{Introducción a la Inmigración a Israel}

El criterio predominante para la ciudadanía en Israel es "jus sanguinis" (Ben-Eliezer, 2004). Es decir, existe un determinismo cultural, compuesto por factores etno-culturales diferencialistas (Brubaker, 1992), que corresponden a una comunidad exclusiva, cerrada que implica un sentido de pertenencia al estado de manera primordial, es inherente -bajo concepciones de ADN- a una determinada persona que cumpla con ciertas características. Bajo esos estándares se define quién tiene acceso a la ciudadanía israelí y quién no.

En este caso, continuando con lo planteado por Brubaker, el concepto de inmigración a Israel es determinante, habla de lo que uno es y la forma en cómo uno se define a sí mismo. Es decir, la ciudadanía a nivel simbólico representa una diferenciación.

El término utilizado en hebreo para llamar a la inmigración judía a la tierra de Israel es "Aliyá" (en hebreo: ascenso), el cual corresponde a uno de los preceptos del judaísmo. La acción inversa, es decir, la emigración desde Israel hacia la diáspora, se conoce por Yeridá que en hebreo significa "descenso".

El concepto de "Aliyá" a la tierra de Israel, es central en la cultura y la religión judía, constituye la base fundamental del sionismo. La Ley de retorno de 1950, que garantiza a cualquier judío del mundo su derecho de inmigrar a Israel, establecerse y obtener la ciudadanía israelí, se basa en dicha idea. 
Durante la historia reciente del pueblo judío, la época del sionismo, hubo cinco olas migratorias masivas antes del establecimiento del Estado de Israel. Luego de la Independencia del Estado de Israel, en 1948, la población judía del país se multiplicó más de ocho veces (gracias a las sucesivas Aliyot), pasando de 650.000 personas en 1948 a unas 5.415 .000 en el 2007 aproximadamente.

\section{Inmigración en tiempos de globalización}

Si se observa el desarrollo del mundo, las olas migratorias en general y el cambio que se ha generado en ese aspecto durante las últimas décadas, a consecuencia de la globalización, que trajo consigo significativas transformaciones a nivel mundial tanto en lo económico, político, cultural, social, legal y comunicacional, principalmente; es posible distinguir que este fenómeno ha generado un cambio que ha reconfigurado no sólo el flujo migratorio, sino también el escenario transnacional y las modalidades de integración a la sociedad receptora. En consecuencia, es posible observar que la mayoría de los países desarrollados se han convertido en una sociedad diversificada, multiétnica, de alta rotación o movilidad en el tiempo, y la tendencia muestra que el resto de las naciones van en ésa dirección (Massey, 1993).

Como consecuencia de lo anteriormente señalado, es notorio el aumento de comunidades transnacionales, las cuales se definen como aquellas comunidades en las que las personas que las conforman, mantienen procesos de identidad en los que relacionan sus lugares de residencia con sus lugares de origen. Estas personas establecen vínculos con dos o más naciones a partir de interacciones económicas, políticas y socioculturales (Portes, 1999).

Las redes transnacionales y los flujos culturales permiten una circulación de forma cómoda, expedita y constante que generan el acceso y el mantenimiento del contacto de los inmigrantes con sus países de origen. Por lo tanto, la etnicidad se mantiene a diferencia de los antiguos inmigrantes. 
Transnacionalización se entiende como el proceso social en el que los inmigrantes establecen campos sociales que cruzan geográfica, cultural y políticamente. El set de identidad se construye derivado de un modelo de ensanchamiento global que envuelve su hogar, la sociedad receptora, así como también otros centros co-étnicos que residen como elementos centrales de la socialización (Glick Schiller et al. 1992).

Los nuevos inmigrantes combinan diferentes repertorios culturales; el prevalecimiento de nuevas opciones étnicas representa caminos a través de los cuales los inmigrantes pueden reconstruir una nueva identidad y así desarrollan una forma de pertenecer a la sociedad receptora.

Además de eso, como la inmigración crece en prevalencia dentro de la comunidad, cambia valores y percepciones culturales de tal manera que incrementa la probabilidad de futuras inmigraciones. Entre los mismos inmigrantes, la experiencia en sí cambia gustos y motivaciones (Piore, 1979). Posterior a la inmigración, los inmigrantes adquieren un fuerte concepto de movilidad social, por lo tanto, se genera la probabilidad de que el inmigrante vuelva a desplazarse nuevamente (Massey, 1986), no necesariamente a su tierra de origen.

\section{Inmigración a Israel en la actualidad}

El caso de la inmigración a Israel no ha quedado exento a estos cambios. En sus inicios, la inmigración a Israel era percibida como una decisión de vida, es decir irreversible. Esto se debía por una parte a las razones que llevaban a la persona a inmigrar a Israel, en complemento con la asistencia que recibía por parte del Estado y la Ley de Absorción; así como también las dificultades de comunicación y movimiento, de menores posibilidades en esa época.

En el caso particular de la inmigración actual a Israel, se distinguen cuatro tipos de motivaciones (Gitelman, 1982):

- Motivación personal: la búsqueda de aventura, de un retiro tranquilo, de reunificación con la familia, de pareja, de trabajo, principalmente. 
- Motivación religiosa: categoría que frecuentemente se mezcla con la categoría que viene a continuación.

- Motivación ideológica-sionista.

- Motivación afiliativa-organizacional: en la cual la persona tal vez socializó en el entorno o en la organización formal en la que inmigrar a Israel es "lo que hay que hacer" y que la inmigración, por lo tanto racionalizada en términos ideológicos, resulta primeramente de conformidad al comportamiento pensado como deseable.

Si bien en la actualidad la referencia a la inmigración a Israel sigue siendo "Aliya", el proceso continúa desarrollándose bajo los estándares de la Ley de Retorno de 1950. Incluso, considerando las razones de inmigración que pueden corresponder a "push factors" ya sean crisis económicas, regímenes políticos, etc., están de igual forma los "pull factors" como por ejemplo razones ideológicas o los beneficios que el inmigrante recibe. Estas son de suma relevancia, el ethos judío-israelí como eje central se relaciona directamente con las razones ideológicas que se encuentran detrás de algunos migrantes a Israel.

Sin embargo, hoy por la forma en cómo marcha el mundo y la manera en cómo funciona el ser humano, la inmigración a Israel ya no es definitiva, es más bien un traspaso de lugar en lugar, es decir, inmigración en tanto cuestión transnacional y multilocal. Desde esa perspectiva, el inmigrante representa una oportunidad en tanto constructor de puente entre culturas (Levitt, 2007).

Los inmigrantes presentan un lazo cercano con sus países de origen y fuertes vínculos con otros coétnicos mundiales, con los que participan en una cultura global. Se aprecia un culturalismo que se relaciona con el concepto proveedor, que precisamente corresponde a uno de los idiomas a través de los cuales la intervención de más y más actores en el sistema internacional, que interactúen entre ellos, más que un modo de malentendido o conflicto (Bayart, 2005). En ese sentido estamos frente a un nuevo marco en cuanto a la inmigración a Israel, que desafía el concepto tradicional de que la Aliyá comprendido como el hecho de que sus inmigrantes son judíos que "vuelven a su patria", ya que además 
desarrollan y mantienen múltiples relaciones familiares, económicas, sociales, organizacionales religiosas y políticas que expanden fronteras (Glick Schiller et al. 1992) y que vienen para quedarse.

La migración actual forma parte del ensanchamiento transnacional de comunidades imaginarias o reales que ofrecen identificaciones alternativas que no eran válidas en los primeros años de la construcción de la nación bajo las políticas de la "hegemonía sionista".

Si bien hoy sigue siendo "Aliyá", es errado considerarlo como la "Mitología de la Unicidad", pues los inmigrantes israelíes manifiestan los mismos atributos que inmigrantes a otros países. En consecuencia, estos conceptos cuestionan el sionismo, la inmigración a Israel y la ciudadanía israelí (Shuval y Leshem, 1998). Esto mismo puede conllevar a que haya más Yeridá, por la misma globalización y expectativas fuera de Israel.

Esto también se observa en la manera en cómo se transfirió de forma simbólica del concepto "Crisol de Razas" (Glazer N. Moynihan D, 1963) a un "Multiculturalismo Étnico". En la práctica, se ve reflejado el cambio en la forma en cómo se relaciona el inmigrante con su nuevo entorno, en comparación a como se relacionaba el inmigrante hace 30 años atrás en la misma situación. En la era de la reactivación étnica, los inmigrantes reformulan sus nuevas etnias y adquieren múltiples identidades que no podrían haberse desarrollado bajo el modelo hegemónico de una única cultura nacional, como el modelo del Crisol de Razas (Anteby-Yemini, 2004).

En la actualidad se distingue un proceso de incorporación, adaptación donde el inmigrante se ajusta a la sociedad receptora, su cultura e instituciones, lo cual se define como un proceso de aculturación (Soysal, 1994). Básicamente, es el término usado para definir el proceso por medio del cual el inmigrante se convierte en un ser absorbido en la población nativa, a través de la convergencia de valores culturales y rasgos personales, así como factores también económicos y ocupacionales de la corriente dominante. Conceptos claves como actitudes, marcos de referencia, motivación social, participación del ego, 
creencias, grupos de referencia, expectativas de rol, comportamiento de rol son considerados como aspectos centrales de inmigrantes y su asimilación a la nueva cultura (Taft, 1957).

Es decir, hoy la persona puede vivir en Israel y mantener su "Argentinidad" o "Chilenidad", en adición a muchas otras identidades que tiene a su elección. El juego interno entre el contexto local israelí, la sociedad de formación de origen y la dimensión global del mundo cultural no puede ser vista como un signo de falla de absorción, si no lo contrario, puede más bien representar una nueva forma de participar en la sociedad israelí que combina nacional, transnacional e identidades globales (Anteby-Yemini, 2004).

En ese sentido, se ve la integración como un proceso de interacción social de ambas partes, y cuando los integrantes de la mayoría comunitaria al igual que los inmigrantes y las minorías étnicas son requeridos para hacer algo, los últimos no pueden ser los únicos culpables de fallar o no tratar de integrarse. La sociedad establecida es el sitio de las instituciones -incluido empleadores, sociedad civil y gobierno- en el cual la integración tiene que tomar un espacio y ellos de acuerdo a eso deben manejarlo (Moodod, 2007). Como es posible imaginar, naturalmente esta "adaptación" viene aparejada con un proceso de cambio que se genera en el inmigrante, lo cual se analiza en términos teóricos a continuación.

\section{Cultura y Adaptación}

El estudio de la cultura es indispensable para una apropiada comprensión de la identidad humana, considerando que los seres humanos son entes en continuo y dinámico desarrollo, al igual que los grupos sociales, las comunidades y las instituciones en las que se encuentran. El escenario que exploró esta investigación, en primera instancia se caracterizó por un cambio profundo en la vida de la persona, el cual se produce en el momento de su inmigración. A consecuencia de esto, el inmigrante se ve enfrentado a cambios y cuestionamientos constantes en torno a su identidad; donde ve alterada su propia consonancia. Su conducta social y sus emociones se ven absolutamente involucradas en todo este oscilante proceso, incluso considerando el pluralismo multicultural del entorno. 
Cabe señalar, que el entendimiento de la cultura en sí misma es una afirmación subjetiva y su fuente de análisis es infinita. Debido a que no existe un concepto absoluto que defina cultura, la forma en cómo el ser humano entiende la cultura difiere respecto al entendimiento del otro.

La proposición que presenta Boesch (1997), puede ser considerada como un modelo estructural del pensamiento sobre la forma en cómo el ser humano entiende la cultura y, en consecuencia, desarrolla la suya. Las definiciones del autor representan una base común que si bien difiere de persona en persona, encausan la forma en que la cultura de cada uno se reconstruye y los factores básicos de cambio que la generan son similares. El autor se refiere a la cultura como sistema de referencia, es decir, cada ítem o evento en un campo cultural, se relaciona con otros ítems o eventos. A su vez, cada evento, objeto o persona está insertado en una complicada red cultural. Por supuesto que nadie está consciente de esto y la cultura para cada persona se centraría en sus áreas de experiencia.

En ese sentido, vemos como en el caso de los integrantes de esta muestra, el ethos judío como parte inseparable de su identidad en la diáspora, representa un referente cultural determinante, de diferenciación y relevancia en sus vidas. En muchos casos, la consecuencia de esto se ve representada por la inmigración a Israel propiamente tal. Si bien de igual forma, tienen toda la cultura y formación de sus países de origen, lo cual también es un repertorio central en sus vidas, se observa cómo esta dualidad de repertorios les provoca crisis de identidad, tanto en la diáspora como en Israel, como consecuencia de las diferentes experiencias que viven.

Boesch se refiere a la acción propiamente tal, la cual es tomada como la búsqueda de un objeto que apunta a un estado futuro del individuo y su situación. Estos objetos están predeterminados y aparecen por diferentes motivaciones, por lo tanto son polivalentes, prometen satisfacer distintas expectativas, incluso la representación de la idea en sí misma puede ser más importante que su resultado. De esta manera, mientras puede ser fácil definir un objeto por su resultado concreto anticipado, es mucho más difícil especificar de forma exacta el significado para el actor. 
En relación a lo anterior, y ejemplificándolo específicamente en el encuentro entre las culturas, el encuentro en sí es complejo, ya que los códigos culturales que poseen sus protagonistas difieren de los del otro, y si la forma en cómo se comunican no fluye, se generan conflictos. Como consecuencia de lo mismo, es que el inmigrante atraviesa por crisis ya que en el nuevo escenario en el que se encuentra, es él quien debe estar abierto a adaptarse a los repertorios culturales de los actores locales, en ese sentido la relación que se genera es jerárquica, ya que el actor local está de manera simbólica en un nivel más alto que el inmigrante. El significado que tiene para él algún tipo de práctica u objeto, para el otro representa algo diferente, incluso y sin ir más lejos, hay actos en el hacer progresivo que para una cultura son percibidos como inherentes y para otra son absolutamente desconocidos, no existen como tales, y vice versa.

La representación de la acción en sí misma puede ser más importante que su resultado, y nuevamente tendrá un valor emocional inconmensurable con características concretas. De igual forma, cabe considerar que toda realización transforma la situación, así es que la acción también impacta en más acciones y sus valencias. Por lo tanto, acción-anticipación también se refiere a otros y puede ser funcional, material, situacional, social.

En el concepto de acción, cada aspecto también hace referencia a otros. Su calidad simbólica deriva de tres sistemas de referencia: cultural, biografía, anticipativo. Esto implica, por supuesto, que ese simbolismo no permanece estático, puede cambiar en mayor o menor medida en el transcurso de la vida, debido a las experiencias personales, al encuentro entre las culturas, la influencia de éstas, sobre todo considerando la era de la globalización, y la forma en cómo el mundo se mueve hoy en día. La visión de la cultura a nivel individual se expande, difiere. La experiencia de vida se enriquece, la acción anticipada varía, todavía en la interacción continúa el ciclo "cultura-individuo-cultura", el cambio será controlado por la necesidad de constancia. 
De esta manera, una acción siempre será realizada por una persona que se beneficia de sus oportunidades o facilidades culturales, y es limitado al mismo tiempo por sus restricciones. El actor seleccionará y adaptará contenidos culturales y, de ese modo creará un significado idiosincrático de la acción. La cultura familiar es su mundo originario, el cual está asociado cercanamente a la familia o con los otros significativos. Las tareas no escritas de cada entorno cultural son organizar, integrar y mantener los patrones psicológicos del individuo ante todo en los años de su formación temprana (Sapir, 1937).

En el caso del inmigrante que se encuentra evaluando de forma constante lo que recibe o rechaza de la cultura local; a través de la interacción continua con varios aspectos del entorno cultural; el sistema interno del individuo experimenta una progresión de cambios cualitativos y cuantitativos por la integración de conceptos culturales aceptables, actitudes y acciones. De esta manera, el individuo encaja para vivir en la compañía de los otros, se adapta a su alrededor, son obligaciones culturales a través de años de socialización (Kim, 2001).

A menudo sucede que el inmigrante, en tanto actor, seleccionó cierta práctica y la adaptó de forma consciente. Otras veces, sucede que lo hace de forma inconsciente, y sólo una vez que percibe que realiza cierto tipo de acción, asume que es parte de él, es decir, durante su proceso de adaptación, va adquiriendo hábitos y prácticas de la cultura local. Es interesante analizar que en este aspecto, el inmigrante atraviesa un shock, que no necesariamente se relaciona con el hecho de tener que lidiar con una cultura diferente, sino en reconocer como su propia cultura lo ha formado, es decir, se da cuenta de la extensión de su cultura en términos de influencia. Y en muchos casos esto también se debe no a la nueva cultura, sino al cambio en los roles que el inmigrante debe asumir en términos personales, sociales o de estructura organizacional y su adaptación a su nueva situación. Como funcionan nuevas culturas o como se ve a sí mismo en nuevos entendimientos, elementos o visiones (Tyler, 1987).

Como consecuencia de este proceso, en ésta "eterna negociación", que incluye todos los cambios que competen a la adaptación del inmigrante; el asumir hábitos y conductas concernientes a la nueva cultura, 
así como también de forma paralela el asumir repertorios fuertemente arraigados en su identidad; es donde se refleja poco a poco el inmigrante asume que va siendo parte de la cultura que lo recibe.

En este proceso, los individuos adquieren afiliación cultural a través de lo cual encuentran un lugar en la sociedad. En cada cultura las personas están conectadas unas con otras a través de un sistema común de codificación y decodificación. Las culturas varían en idioma y comportamiento verbal, modales y movimiento, maneras de sentarse, pararse, manejar el tiempo, espacio y materiales. Es la cultura la que programa a los miembros de una sociedad a interpretar mensajes verbales y no verbales definiendo lo que es real, verdadero, lo que está bien, qué es lindo y qué es bueno. Situaciones culturales condicionan a los individuos a ciertos patrones de pensamiento, sentimientos y comportamientos en variadas transacciones sociales. La cultura como patrón de percepciones, actitudes y comportamientos que son aceptados y esperados por otros en una determinada sociedad más allá del nivel de consciencia pensado (Kim, 2001).

En consecuencia, el proceso por el que atraviesa el inmigrante, implica el desarrollo individual de la habilidad de organizar sus actividades con las de otros en la sociedad. Esa habilidad, muchas veces llamada "competencia social" (Kim, 2001), comprende aspectos cognitivos, afectivos y operacionales (de comportamiento); capacidades a través de las cuales los individuos se organizan a sí mismos en y con su entorno socio cultural.

Así como los individuos incorporan patrones culturales en su psique, también desarrollan identidad cultural. A través del desarrollo de competencias comunicacionales y funciones idóneas, internalizan patrones culturales, que se convierten en su mundo con una fuerte protección de matiz emocional.

La adaptación y el cambio que esto conlleva, inevitablemente causa stress en la psique de los inmigrantes, una disonancia que genera un conflicto entre el deseo de retener viejas costumbres y mantener su identidad original por un lado, y el deseo de adoptar nuevas formas para buscar armonía 
con el nuevo entorno, por otro lado. El conflicto es esencialmente entre lo que Kim (2001) define como la necesidad de aculturación y la resistencia a la deculturación. El "Pull" de la nueva cultura y el "Push" de la antigua. Entre las condiciones existentes dentro del inmigrante y las demandas del ambiente externo.

\section{Cruce de culturas}

Todas las personas que cruzan o atraviesan culturas, se enfrentan a algunos desafíos comunes, pioneros en desarraigarse de sus raíces y al establecimiento de relaciones graduales laborales y sociales con su nuevo entorno.

El espacio entre lo familiar y cómodo de los alrededores de sus hogares y lo poco familiar del ambiente que recibe, limita la habilidad de funcionar de manera efectiva. Muchos de los modos de comportamiento útiles en el antiguo marco, pueden provocar una mala adaptación en el nuevo marco. Reconocimiento de códigos verbales y no verbales e interpretación de las asumiciones escondidas, se acentúan como dificultosas (Kim, 2001).

Algunos resistentes al cambio, pelean por sus viejas formas, mientras otros, tratan de forma desesperada de ser nativos y vivir con una sensación aguda de fracaso y desesperación y su entorno inmediato influirá definitivamente en ello. Diferentes razones de atravesar culturas, acompañan grados de compromiso que los individuos sienten frente al nuevo entorno.

El proceso de atravesar culturas, desafía la base de quien es uno en tanto ser cultural. Ofrece oportunidades de nuevo aprendizaje y crecimiento. Ser desarraigado de su propio hogar trae entendimiento no solo de las personas y su cultura en el nuevo ambiente, sino también de uno mismo y su cultura natal (Kim, 2001).

Adaptarse a una nueva y desconocida cultura, es más que supervivencia, un viaje de cambio de vida, es también independencia y un viaje a la madurez. Es de alguna forma empezar conscientemente una vida de nuevo, con todo lo que eso implica. 
Cuando personas extrañas entran a una nueva cultura, el proceso de adaptación al cruce cultural es un hecho en marcha. Este proceso continúa así como la persona mantenga algún tipo de comunicación con el entorno anfitrión.

A través del proceso, el individuo experimenta un grado de transformación intercultural correspondiente a una extensión de su comunicación e interacción con el entorno anfitrión. La adaptación ocurre natural e inevitablemente incluso cuando los extraños no la planean o activan buscando para que esto ocurra, aunque no tengan intención de participar completamente en las actividades comunicacionales sociales de la sociedad anfitriona, y se confinen a sí mismos casi en su mayoría a relaciones superficiales con los nativos. Los cambios de adaptación ocurren gradualmente, casi imperceptiblemente. Sin perjuicio de lo anterior, la globalización, la comunicación, el Internet, han facilitado el proceso de conectarnos con otras culturas, lo que nos ha iniciado en este cruce intercultural.

\section{De una identidad cultural a una identidad intercultural}

La identidad cultural se refiere a una definición de uno mismo por los otros que sirve para marcar o referirse a un sistema de conocimiento y significado, una extensión de horizonte conceptual contra la cual el individuo evalúa sus propios pensamientos y acciones.

En este proceso, la identidad se puede definir como un proceso de evolución continuo, que se mueve en dirección hacia una completa integración psicológica mental.

Adler (1982) define que el desarrollo de una identidad intercultural sitúa al extraño en una posición de continua negociación con nuevas formaciones de la realidad. En términos prácticos, así como la identidad cultural vincula a una persona con una cultura específica, la identidad intercultural conecta a una persona con más de una cultura. 
Se aspira a que el inmigrante al desarrollar su identidad intercultural haya internalizado un incremento en el nivel de competencia comunicacional de la cultura que lo absorbe y una integración en el proceso social de comunicación del anfitrión.

En este caso, los inmigrantes están más capacitados para manejar la dinámica de la interacción del diálogo entre la cultura original y la nueva cultura. También poseen mayor capacidad de experimentar diferentes mundos culturales con más facilidad, tienen de igual forma mayor capacidad de tomar decisiones deliberadas de acciones en situaciones específicas, más que simplemente seguir los dictados de las normas prevalecientes de la cultura de su niñez.

La adaptación del cruce cultural representa un viaje personal que conduce la categoría de trascendencia cultural en una consciencia individual. Convertirse en intercultural no significa reemplazar una cultura por otra, si no "trabajar a través de" todas las experiencias culturales para crear nuevas construcciones. Esto va asociado al aprendizaje, pues asumir nuevas experiencias es parte del proceso de maduración del individuo enfrentado, sólo, a una nueva cultura a la que debe incorporarse.

En el cruce de culturas, la mezcla de contactos culturales toma muchas formas, los actores se comportan de acuerdo a las normas del grupo al cual pertenecen y se adscriben al objetivo de personas con cualidades que se supone son características a ese grupo de personas. Personas que pertenecen tanto al "nosotros" (mi familia, mis amigos, mi país) y al "ellos" (desconocidos, enemigos, extranjeros). El prerequisito para un contacto intercultural exitoso es que ambas partes deben ser sensibles al impacto que tienen sobre el otro, es decir, "outsiders" menos intrusivos e "insiders" menos defensivos (Bochner, 1992).

Para borrar la distinción entre "ellos" y "nosotros", las personas deben convertirse parcialmente en "nosotros", es decir, incorporar algunas de "sus" características sin perder su propia identidad étnica. 
En ese sentido, el desarrollo de una personalidad o identidad intercultural es el último resultado del proceso de adaptación del cruce cultural (Kim, 2001).

\section{Comunicación y Lenguaje}

La Comunicación es el pilar fundamental de todo aprendizaje humano. El ser humano aprende a hablar, escuchar, leer, interpretar y entender mensajes verbales y no verbales de una forma en la que el individuo y los otros seres con los que interactúa pueden reconocer, aceptar y responder a esos mensajes. Una vez adquiridas, las habilidades comunicativas sirven como instrumento interpretativo y expresivo, lo que significa llegar a términos con el ambiente propio de cada uno (Kim, 2001).

Cada persona tiene actitudes emociones, prejuicios, hábitos y peculiaridades que son tan parte de él, así como el lenguaje que habla, o las creencias que acepta o reacciona hacia ellas regularmente. Los cambios en las personas, tienen consecuencias también en los otros (Tyler, 1987). Aprender a ajustar y reajustar es parte de la vida, especialmente con los variados significados interculturales que esto implica, según lo visto anteriormente.

Se puede pensar que saber usar el idioma de las personas del nuevo entorno que rodea al inmigrante, asegura una automática comunicación con ellos. Pero la comunicación efectiva incluye todo, el comportamiento y las circunstancias. El conocimiento de un idioma hablado y escrito es en sí mismo insuficiente.

La efectividad en la comunicación depende de miles de signos y símbolos de los que la persona es en gran parte inconsciente. Estos signos e indicaciones que la persona utiliza para orientarse a sí mismo en situaciones cotidianas, incluyen por ejemplo cuándo y cómo saludar a alguien, como comer, como moverse de lugar en lugar, como hablar informalmente con otros, los gestos, cuándo hablar y cuándo no (Tyler, 1987). 
Cuando la persona entra en contacto con una cultura extraña, todas o la mayoría de las indicaciones familiares se remueven y eso puede hacerlo sentir como un "pez fuera del agua". La dificultad en la confrontación y la copia de las nuevas circunstancias culturales, se definen como "shock cultural" (Tyler, 1987), y éste afecta a casi todos los inmigrantes. Por supuesto que los grados de influencia de la afección son relativos de persona en persona, en consecuencia hay algunos individuos que se adaptan más rápido que otros.

En el caso particular de esta investigación, resalta como característica importante en términos de identidad, lenguaje y comunicación el tema del idioma, ya que en Israel el idioma oficial es hebreo. Considerando el lenguaje como el instrumento que nos permite compartir nuestra experiencia del mundo con otros seres humanos, creando "nuestras-relaciones" (Shutz, 1967), en relación a lo anterior, se ve el idioma como un aspecto problemático dentro de la inmigración. Esto se genera producto de que el idioma representa una llave de entrada primordial en términos de inserción socio cultural, académico y/o laboral. Por esta razón, muchas veces el inmigrante ve en el idioma su principal barrera de entrada y en consecuencia, lo percibe como una pérdida, sobre todo en la primera etapa de la inmigración.

Continuando con lo anterior, se ve como el idioma tiene directa relación con la inserción laboral, ya que el trabajo al que va a poder acceder una persona que maneja el idioma se diferencia mucho al tipo de trabajo al que va a poder acceder un inmigrante que no tiene conocimiento de éste. En consecuencia, el trabajo se percibe como algo más profundo que una entrada económica, éste se relaciona con un proceso simbólico y representa un tipo de status (Piore, 1979).

Esto se ve reflejado directamente en los casos que pertenecen a la investigación, y en los trabajos que desarrollan los inmigrantes cuando recién llegan al país, sobre todo considerando que en la mayoría de los casos pertenecientes a la muestra, deben combinar estudios con trabajo. Por lo tanto, el tipo de status que le gustaría tener al inmigrante en relación al área laboral, dista mucho de lo que está desarrollando o 
puede desempeñar en ese período. Sin ir más lejos, en muchos casos, el inmigrante se ve desarrollando trabajos que en su país de origen no habría realizado porque éstos no son bien vistos.

Por otro lado, en estos casos donde el inmigrante debe estudiar y trabajar para poder mantenerse, el trabajo pasa a ser un medio que le permite poder cumplir con su objetivo en términos académicos. En consecuencia, posterga su inserción al mercado laboral profesional durante ese período, aspirando a poder lograr ese status, y subir en esa categoría que en definitiva y en conjunto con el idioma representan una absorción exitosa.

\section{Inmigración Latinoamericana a Israel}

En la era del pluralismo multicultural, no se ve asimilación, si no una incorporación donde el inmigrante mantiene su lengua nativa. En la actualidad y llevado al caso de la inmigración latinoamericana a Israel, se ve cómo los medios de comunicación han emergido y son agentes importantes que contribuyen al pluralismo. Si bien en comparación a las inmigraciones etíopes o rusas la presencia en los medios es menor, esto se atribuye a dos razones: en primer lugar en términos proporcionales las olas migratorias latinoamericanas han sido siempre constantes, la diferencia es que se realizan en contingentes pequeños desde los años 40 (Friedlander y Goldscheider, 1984); en segundo lugar, tomando como referencia la definición de los latinoamericanos en Israel, como una "Comunidad Invisible" (Roniger y Jarochevksy, 1992), la presencia es en tanto menor.

El término de "comunidad invisible" se atribuye directamente a la rápida incorporación que tienen los inmigrantes latinoamericanos en la sociedad israelí, en comparación a otros grupos de inmigrantes. De todas formas cabe señalar que esta invisibilidad no ha sido unánime ni uniforme. La voluntad de los inmigrantes latinoamericanos de perder su "visibilidad" al incorporarse al resto de la sociedad receptora, se relaciona directamente con la naturaleza del judaísmo latinoamericano, donde la educación judía y la vida en torno a la comunidad, son ejes centrales en el crecimiento, formación y desarrollo del judío que vive su "identidad dual" (Roniger y Jarochevksy, 1992), judío-argentino y/o judío chileno -en el caso de 
este estudio- en esos lugares. En complemento a las razones ya sean ideológicas, económicas, religiosas, entre otras, que han llevado a los miembros de esas comunidades a radicarse en Israel.

En este punto se puede definir que estamos frente a casos de "Core Jews" que son aquellos cuya identidad, afiliación e identificación es claramente judía, lo que también se traduce en una fuerte conexión con la patria. No es del todo claro si esto está basado netamente en creencias religiosas o seculares, ideológicas, visiones o sentimientos etno-nacionales. Lo que sí queda claro es que es de su interés asegurar la continuidad judía. Así como también su identidad e identificación están basadas en una visión comunal etno-nacional y sus sentimientos están basados en principios culturales universales, más que construidos sobre una base de elementos religiosos únicamente (Sheffer, 2007).

Es esa identidad e identificación que poseen los "Core Jews" la que representa los elementos más significativos que determinan la participación activa en entidades judías.

En este sentido se podría decir que en la inmigración a Israel por parte de los latinoamericanos, existe una elección ideológica, ya sea sionismo y/o judaísmo, que representan los motivos dominantes de por sí en la decisión de la inmigración (Gitelman, 1982), lo cual se relaciona con ideales de vida, identidad personal, incluyendo la identidad judía que quieren para sus hijos, que se complementa con la elección demográfico-económica. Al inmigrar a Israel, los migrantes han pretendido mantener en armonía su idiosincrasia judía con los valores humanistas y universales que muchos profesaban. Los migrantes ven su inmigración a Israel como un acto de plena voluntad (Roniger y Jarochevksy, 1992). En ese sentido, la inmigración a Israel se percibe como "un acto de llegada a..." y no como "una escapada de..." (Gitelman, 1982).

El contingente de inmigrantes latinoamericanos, constituye un grupo con características propias, que se mantienen estables a lo largo de los años. En general, llegan a Israel bien preparados en términos de conocimiento de Israel, teniendo formación tanto judía como sionista, así como también del idioma, 
(Roniger y Jarochevksy, 1992) -característica relevante anteriormente analizada-, estos conocimientos fueron adquiridos en el colegio, movimientos juveniles, en complemento con sus hogares y la vida comunitaria en sus países de origen en general. Esto se debe netamente a la "cultura corporativa", bajo la cual funcionan las comunidades en cuestión, que contienen los caminos comunes en los cuales sus miembros aprenden a pensar, sentir y actuar (Hoftsede, 1997).

Como una clara consecuencia de esto, respondiendo a la formación que recibieron los integrantes de esta muestra específicamente, la mayoría de estos ya conocían el país, habían estado de visita o permanecido por períodos más prolongados, anteriores a su establecimiento en éste con status de ciudadanos. Por lo tanto, inmigraron con cierta noción del lugar, aunque claro está, el hecho de estar como turista dista mucho de la realidad que se vive en tanto inmigrante. El valor que tiene el hecho de vivir en el lugar como un ciudadano más, por así haberlo elegido, representa un capital simbólico único para el inmigrante que marca la constitución de un proceso en desarrollo de construcción de la nueva realidad que lo rodea.

\section{Metodología y Muestra}

Por las características de esta investigación, la técnica más adecuada para la recolección de la información fue entrevistas en profundidad. Las cuales se complementaron con una observación participante lo que permitió completar el seguimiento de los integrantes de la muestra, una vez concluido el periodo de las entrevistas, antes de haber cumplido tres años viviendo en Israel.

El grupo de entrevistados se compone por nueve jóvenes entre 20 y 35 años de edad, provenientes de Argentina y Chile que inmigraron a Israel durante el año 2005. Los nueve integrantes que componen el estudio, inmigraron a Israel bajo la Ley de Retorno y recibieron los derechos de inmigración y asistencia, así como la ciudadanía israelí de forma inmediata.

Las entrevistas fueron realizadas durante el primer semestre de 2007, período en el cual los entrevistados aún no cumplían dos años viviendo en Israel. La muestra está compuesta por cuatro 
mujeres y cinco hombres, de los cuales son todos solteros sin hijos, todos judíos de origen, salvo una entrevistada conversa al judaísmo en su país de origen. La muestra a su vez se caracteriza por un grupo de estrato socio económico variado, dentro de esa heterogeneidad la gran mayoría pertenece a un estrato social medio, con un nivel de educación primario y secundario, donde todos pasaron por educación superior aunque al momento de inmigrar no todos habían alcanzado un titulo técnico o profesional. Un rasgo relevante en la composición de la muestra es la ideología que caracteriza a los integrantes, la cual se manifiesta en su visión sobre Israel, sionismo y judaísmo como repertorio relevante en tanto forma de vida en términos culturales.

El esquema de las entrevistas se dividió básicamente en dos partes. En primera instancia, se indagaron los motivos por los cuales el joven decide inmigrar a Israel, y de igual manera su estilo de vida y entorno en su país de origen. En segunda instancia, se indagó en la vida del joven una vez estando en Israel. Es decir, la forma en cómo vivió el proceso de cambio inicial, el choque cultural, el encuentro con la nueva cultura, su crisis de identidad, su adaptación, su aculturación, su percepción del nuevo entorno, la cultura israelí y la sociedad receptora en general.

\section{Conclusiones e hipótesis}

Al momento de concluir la investigación, el proceso de inmigración de los integrantes de la muestra aún estaba en pleno desarrollo. Por lo tanto, sus percepciones, estados de pertenencia, rechazos, incorporaciones, así como gustos y preferencias varían según la etapa por la que cada uno esté atravesando en el transcurso de inserción al país.

La inserción y el sentirse más establecido en el país varía de inmigrante en inmigrante, tiene relación directa con su formación, su personalidad, su esencia, sus aspiraciones y la forma de ser del individuo en sí, sus logros y expectativas. Esto por cuanto la adaptación es un proceso personal en el cual el individuo responde a estímulos externos de manera diferente del otro, en tanto ser único. A pesar de eso, son observables características comunes en cuanto al proceso. 
Cabe señalar que de todos los integrantes de la muestra en análisis, hay un integrante que durante el proceso del estudio se encontraba preparando el regreso a su país de origen. El resto de los entrevistados aún permanecen en Israel, con sus planes en desarrollo así como señalan en sus entrevistas.

\section{Identidad y Cultura}

Para los integrantes de la muestra, Israel es percibido como un país joven, con una sociedad joven, que se encuentra en proceso de crecimiento con todo lo que eso implica. A causa de la forma en cómo se ha desarrollado la historia de éste, los conflictos con los países vecinos, se vive una sensación de inmediatez e incertidumbre bastante particular. Es una característica intrínseca del país y su sociedad, la cual también es percibida por los investigados, que de alguna forma han integrado esa sensación a sus vidas.

En términos generales, la primera impresión en relación al israelí es que consideran que no respeta el espacio ni la intimidad del otro. Plantean que el israelí cree que todo lo que pasa a su alrededor le pertenece, es frontal en su forma de decir las cosas y muchas veces se expresa de mala manera. Eso les genera rechazo, aunque a su vez, está la percepción de que cuando han necesitado ayuda, el israelí les da una mano y es una ayuda real. Destacan de igual forma, el hecho de que el israelí exprese las cosas como son, lo que no es muy común en sus países de origen.

En relación a la identidad del inmigrante, ambivalente de por sí, es observable que atraviesa por crisis y cambios durante todo este proceso. Sumado a esto, el inmigrante vive en un constante análisis de la nueva sociedad en la que está inmerso y a la cual aspira a pertenecer. En consecuencia, evalúa de forma sistemática aspectos que está dispuesto a ceder, aceptar, modificar, adecuar o rechazar con el fin de insertarse mejor al país. 
En consecuencia, y producto de que el inmigrante proviene de una idiosincrasia diferente a la israelí, éste posee una formación social distinta. En el encuentro con esta nueva cultura para él, va conociendo y adquiriendo hábitos diferentes, muchas veces estos cambios no son percibidos por él, y solamente los advierte cuando finalmente asimiló este nuevo hábito en su vida y lo integró a ésta. $\mathrm{O}$ más aún, en algunos casos se refleja sólo cuando alguien externo le advierte sobre eso; a modo de ejemplo, el comentario más banal que el inmigrante a veces escucha es: "estás hecho todo(a) un israelí" o "estás cada día más israelí".

En relación a la integración a la sociedad y la forma en cómo define el inmigrante ese aspecto, es interesante analizar la importancia que tiene para él el hecho de tener más contacto con israelíes, conocer y sumar amigos israelíes es fundamental. Todos los entrevistados señalaron que eso representa un aspecto que define lo integrados que se sienten de la sociedad o no.

En oposición a lo anterior, Israel tiene la peculiaridad de ser un país multicultural en términos de sociedad. La diversidad y proveniencia de sus habitantes, los cuales en gran parte fueron inmigrantes en algún minuto, concibe una sensación de estar inmerso dentro de un gran "zoológico humano". Vivir en un escenario multiétnico, además del interés que genera en términos de grupo humano, como manifestaron los integrantes de la muestra, en el tiempo también provoca una sensación de pertenencia, es decir, con el tiempo el inmigrante siente que aún con su diferencia de igual manera pertenece a Israel y a su sociedad y que no necesariamente debe relacionarse $100 \%$ con israelíes para que así sea.

\section{Idioma}

El idioma representa un tema central para el inmigrante. Si bien algunos vienen con conocimientos previos y eso facilita de alguna forma su inserción, al momento de entrar en contacto con israelíes en instituciones como por ejemplo la universidad o el trabajo, les produce una sensación de desventaja, muchas veces es una barrera de entrada. 
Durante la primera parte del proceso de inmigración, los investigados invirtieron mucho tiempo y energía con el fin de lograr un nivel adecuado de hebreo que les permitiese interactuar y desarrollarse en diferentes ámbitos de manera adecuada. A pesar de eso, una vez que entran en contacto con la realidad local, muchas veces por no decir la mayoría, sienten que su nivel de hebreo no es suficiente o es inadecuado. Sobre todo en el ámbito académico, como lo expresaron muchos de los entrevistados. En otros casos se da que cuando se encuentran en un ambiente donde la gran mayoría de las personas son israelíes, algunas veces se sienten incómodos, ya que les falta vocabulario para poder expresarse como les gustaría o simplemente porque hay una serie de códigos que aún no manejan.

A pesar de eso, respecto a lo observado desde que se inició la investigación hasta su conclusión, se observó un proceso natural en el cual los inmigrantes incrementaron su contacto con la realidad local. Es decir, manejan más códigos, se pueden comunicar de mejor manera, su nivel de hebreo es más alto, tienen más contacto con israelíes en general. Esto se ve reflejado de igual forma en trabajos que han adquirido o actividades sociales que han ido integrando a sus vidas, por ejemplo pertenecer a un taller de drama, asistir al teatro y comprender la obra, dar recorridos en hebreo a los visitantes del museo, entre otras.

\section{Rango Etario}

Una característica determinante dentro de los integrantes de la muestra es la edad. El rango etario en el momento en el que se concretó su inmigración es fundamental para entender el proceso. En primera instancia y en términos sociales, la edad de alguna manera permite o no permite realizar ciertas cosas. Es decir, es diferente inmigrar sólo a los 25 años de edad que a los 40 años. Esto debido a la forma en cómo se desarrolla el ciclo de la vida del ser humano, la persona asume responsabilidades mayores a medida que pasa el tiempo y tiene distintos plazos para realizar o alcanzar su proyecto. 
En muchos casos la inmigración no se concretó antes por un asunto de edad, ya que los padres no lo permitieron, porque la persona debía cumplir con ciertas obligaciones impuestas como comenzar y concluir sus estudios superiores, principalmente.

Por otro lado, la inmigración se concretó en ese momento ya que la persona se encontraba en un proceso de transición lo cual representaba la instancia ideal para inmigrar ya que después es demasiado tarde, en el sentido de que con el correr del tiempo es más dificultoso concretar la partida. El período de transición, puede graficarse por ejemplo con la finalización de los estudios superiores y el ingreso al mercado laboral que representa una instancia de establecimiento, y hace más compleja la concreción de la inmigración.

En el caso de los integrantes de la muestra, la edad es concluyente en el sentido de que son jóvenes que migraron en un período determinado de sus vidas, que en la mayoría de los casos está determinado por la transición entre la finalización de sus estudios y el ingreso formal al mercado laboral, por lo mismo se encuentran en una especie de transición. En el caso de que quisieran volver a sus países de origen o inmigrar a otro país, ya sea porque no se adaptaron, por buscar otras oportunidades o por un sin fin de otras razones, la edad todavía se los hubiese permitido.

\section{Relaciones Sociales}

En una primera instancia respecto al grupo social que rodea a la muestra, algunos tienen uno que otro amigo israelí, ya sea del trabajo, estudios, ejército, amistades previas, etc., los lazos de amistad que forjan, son principalmente con otros inmigrantes, que están pasando por el mismo proceso de ellos. Naturalmente esto se produce debido al proceso compartido por el que los inmigrantes están atravesando, se crea una especie de red social, donde las relaciones son percibidas como a nivel familia. Esto se genera esencialmente porque los jóvenes inmigraron solos al país, comienzan una nueva vida, partiendo de cero y tienen una necesidad social de relevancia, necesitan respaldo, contención y poder compartir lo que les ocurre con el resto. Además de la etnicidad, que los acopla de manera automática, 
incluso a pesar de las diferencias idiosincráticas de cada país, hay un código común, aparte del idioma, más semejante entre latinoamericanos que entre un israelí y un latinoamericano.

Sin embargo, en la actualidad es observable una mayor apertura y relación con israelíes en general. Planteo mi hipótesis de que es una consecuencia natural de su mayor incorporación a la sociedad en términos generales, es decir, el migrante ya no necesita tanta contención de su "red familiar". Se siente más seguro, está más abierto y en consecuencia, comienza a formar lazos más profundos con israelíes, es decir, en los mismos escenarios en los que interactuaba antes, ahora se va arraigando más en proporción a la primera etapa, se observa más interacción.

Proyectando la hipótesis, es sorprendente ver cómo no se manifiesta rechazo, por ejemplo, hacia formar una pareja con un o una israelí. Es decir, se observa que a pesar de toda la crítica y la impresión que les generan los israelíes en términos generales, por diferencias en su formación social, no existe una repercusión per se en ese aspecto. Los inmigrantes están abiertos a probar, conocer. Si se analiza este planteamiento de manera más detallada, y en relación a la inmigración en sí, en primera instancia esto representa el arraigo del inmigrante en la forma y significación más profunda de la palabra, de alguna manera "renuncia" a su etnicidad, que se mantendría más latente si el inmigrante formara pareja con otro inmigrante latinoamericano. Además de dejar abierta la posibilidad de volver a su país de origen, que con otro latinoamericano representa mucha menos dificultad.

Continuando con la proyección anterior, esto también se debe a que para este tipo de inmigrantes el ethos judío es muy relevante, su inmigración es una consecuencia de eso y si bien es temprano concluir respecto a la proyección de pareja de los integrantes de la muestra, de acuerdo a la forma en cómo se manifiestan y a las relaciones que se han observado en algunos casos, la hipótesis planteada tiene como consecuencia la asimilación étnica. 


\section{Estilo de vida de los inmigrantes}

Respecto al nivel de vida que tienen los investigados en Israel, reconocen que es muy diferente al que tenían en su país de origen. La gran mayoría de los pertenecientes a la muestra, se hizo independiente de sus padres al momento de inmigrar, con todo lo que eso implica, es una madurez obligada, el hecho de asumir independencia, responsabilidad plena y absoluta de sus actos y su vida con las consecuencias que esto trae, la sensación de que la persona está consigo misma y todo depende de él.

Consecuencia de lo anterior, sumado a las características de Israel en particular, el estilo de vida que tienen los integrantes de la muestra, es diferente al que llevaban en sus países. Esto radica principalmente en que los que no estaban haciendo el ejército debían trabajar para mantenerse y los que sumado a eso estudian, también. Además, la vida es más costosa en comparación a su realidad anterior, producto de la independencia asumieron gastos que antes no tenían, es más difícil, el cansancio y la falta de tiempo no les permite desarrollar o hacer todas las cosas que les gustaría. Su economía tampoco se los permite, hay un cambio evidente en las necesidades, sobre todo en una primera etapa, donde todavía no están $100 \%$ asentados. Considerando que la mayoría de los inmigrantes provenía de una clase media, manifiestan un cambio de vida en relación al estrato social, donde pasaron a un estrato más proletario, en alguno de los casos.

Esta percepción que se presenta en algunos casos en relación al descenso en su status social, se relaciona directamente con el hecho de que las sociedades latinoamericanas en general están muy estratificadas, hay relaciones jerárquicas muy marcadas, contraria a la movilidad de la sociedad israelí en ese aspecto, lo que no permite el estilo de vida latinoamericano. En consecuencia, en ese sentido, para algunos inmigrantes esto representó un cambio brusco, lo que también se relaciona con los trabajos a los que el inmigrante accede sobre todo en la primera etapa y sus expectativas futuras.

Una característica importante respecto al estilo de vida, es la percepción de la inmigración como una aventura experimental, que se relaciona directamente con lo planteado anteriormente respecto a la edad. 
La inmigración se puede vivir como una aventura, ya que la edad de los integrantes de la muestra, también lo permite, pero el sentido más profundo de esto es que la aventura y la sensación de libertad que viene aparejada con ella, se genera producto de que en su nuevo entorno el inmigrante es un desconocido, nadie sabe quién es, de dónde viene, a qué familia pertenece, etc.

Como resultado de lo anterior, de forma natural, el inmigrante posee un margen de maniobra mayor que en sus países de origen, lo cual es muy atractivo para él. Esto se debe a que en Israel alcanzó su independencia, pero más allá de eso, está consigo mismo con todo lo que eso implica y no debe rendirle explicaciones a nadie. Esa libertad de acción lo lleva a permitirse desarrollarse en aspectos distintos, probar cosas nuevas, estudiar áreas o carreras distintas.

\section{Inmigración a Israel}

Respecto a la inmigración, planteo la hipótesis de que en primera instancia la inmigración en sí es percibida como una oportunidad por el inmigrante. En el sentido más profundo de su ser, el inmigrante alberga sueños, expectativas y planes, los cuales trascienden a las facilidades que otorga Israel. Esta oportunidad es simbólica e intangible, en el sentido de que la inmigración representa la idea de empezar de cero en otro lugar, está la posibilidad de la aventura de permitirse cosas que en otras instancias no haría. Esto trasciende a las oportunidades que ve el inmigrante en el plan en sí, aunque en la realidad post inmigración, los planes fantaseados no se hayan concretado de manera propiamente tal.

Continuando con la idea de la inmigración en tanto oportunidad, en términos prácticos, si bien en muy poco de los integrantes de la muestra salen a relucir las posibilidades que ofrece Israel en relación a la asistencia económica y ayuda que otorga a los inmigrantes, está intrínseco, y aunque no lo hayan expresado en sí, es un "Pull" factor determinante el hecho de poder acceder a esa asistencia. Es decir, la inmigración a Israel es para los inmigrantes una oportunidad en términos funcionales, como opción económica o de carrera. 
Por otro lado, si se considera a los integrantes de la muestra, un "Push" factor que se ve en la inmigración a Israel en particular es el riesgo de asimilación en sus países de origen. Es un miedo intrínseco para cualquier "Core Jew" que vive en la diáspora.

La inmigración a Israel es una consecuencia de la "carga ideológica" de la mayoría de los casos en cuestión. Se concluye que el trasfondo ideológico comunitario fue determinante en la decisión de inmigrar. Incluso aunque una vez en su encuentro con la realidad local, esa imagen y carga ideológica se modifique y en algunos casos ya no se sostenga como tal.

Por otra parte, la inmigración a Israel por razones ideológicas, como característica común de la gran mayoría de los casos que integran la muestra, en este caso representa una moneda con dos caras. La ideología en su sentido más funcional, desde una arista por excelencia marxista, se ve representada de manera analógica en la moneda que por un lado contiene la función afectiva y en consecuencia rectora que esta le otorga. Por otro lado, está la insostenibilidad de la ideología y por ende la justificación del regreso.

\section{Comentarios finales}

Analizando a los investigados en el tiempo, y al haber finalizado la investigación, se concluye que en los migrantes que integraron el estudio, efectivamente se observa una aculturación, hay una transformación intercultural, la cual se manifiesta en una emergente identidad intercultural que trasciende la identidad cultural original de cada uno. Esto es observable en sus cambios de hábitos, adquisición de nuevas costumbres propias de la cultural local, cambios en sus gustos, etc. los cuales se han asimilado y prolongado en el tiempo. Estas permutaciones conductuales de personalidad y de estilo de vida, son propias de la migración y responde a lo esperado de acuerdo a la etapa que estaban atravesando los investigados en los momentos en los que fueron entrevistados. 
De esta manera se concluye que efectivamente después de inmigrar no hay vuelta atrás, en el sentido de que hay otro fondo, más profundo, en cuanto a la forma en cómo se define la vida, en el cual el inmigrante vive con la complejidad y la riqueza de otra visión.

La inmigración y todo el desarrollo de este proceso en sí, implica un desafío constante de uno mismo, donde la persona se pone a prueba de forma permanente, muchas veces sintiendo que ha llegado a su límite. Pero como al fin y al cabo los límites los va fijando el propio ser humano, el desafío de la inmigración en sí mismo representa una historia sin final, en la cual se van agregando capítulos todos los días, que incluyen más experiencia, crecimiento personal y nuevos retos.

Cabe destacar que al ser un proceso en desarrollo, está propenso a cambios de manera constante. Es decir, las percepciones de lo que hoy define la israelidad y la pertenencia de cada uno de los inmigrantes en cuestión, puede ser diferente a lo que defina su israelidad mañana. Conscientes de eso y habiendo pasado más dos años desde su llegada a Israel, siguen viviendo su absorción como un proceso, que para la mayoría tiene como fin establecerse de forma definitiva en Israel. 


\section{Bibliografía}

ADLER, Mortimer J. Six Great Ideas Macmillan Pub. Co. 1982.

ANTEBY, Lisa, "Being an Oleh in a Global World: from Neo-Ethnic Community to Transnational Community" EN: KEMP et al. Israelis in Conflict: Identities, Hegemonies, Challenges Brighton: Sussex University Press, 2004.

BAYART, Jean-Francois. The Illusion of Cultural Identity C. Hurst and Co. Ltd. Publishers, 2005.

BOCHNER Stephen. Cultures in Contact: Studies in Cross-Cultural interaction. Pergamon Press, 1992.

BOESCH, Ernst E. "Symbolic Action Theory in Cultural Psychology" Culture \& Psychology Vol. 7 (4) Sage

Publications. London and New Delhi, 1997.

BRUBAKER, Rogers. Citizenchip and nationhood in France and Germany Cambridge, Mass.: Harvard University Press, 1992.

FRIEDLANDER, D. and GOLDSCHEIDER, C. Israel's Population. The Challenges of Pluralism Washington, Population Reference Bureau, 1984.

GITELMAN, Zvi. Becoming Israelis: Political Resocialization of Soviet and American Inmigrants Praeger, 1982.

GLAZER N. MOYNIHAN D, Beyond the melting pot: The Negroes, Puerto Ricans, Jews, Italians, and Irish in New York city. Cambridge MA, MIT, 1970 (1963).

GLICK SCHILLER et al. Towards a trasnational perspective on migration: race, class, ethnicity and nationalis reconsidered The New York Academy of sciences. New York, 1992.

HOFSTEDE, Geert. Cultures and Organizations: Software of the Mind. London, McGraw Hill, 1991.

KIM, Young Yum. Becoming Intercultural Sage Publications, 2001.

LEVITT, Peggy. God needs no passport: How to inmigrants are changing the American Religious Landscape The New Press, 2007.

MASSEY, D, ARANGO J, GRAEME H, KOUAOUCI A, PELLEGRINO A and TAYLOR E, "Theories of International Migration: An Integration and Appraisal," Population and Development Review 19: 431-466, 1993. 
MERKX, G., LAIKIN J. (Eds). The Jewish Presence in Latin America Boston, Allen \& Unwin, 1987.

MODOOD, Tariq. Multiculturalism, a Civic Idea Polity Press, 2007.

PÉREZ Serrano, Maria Gloria. Research - Action, Aplications to the social and educational field Dykinson Editorial. (In Spanish), 1990.

PIORE, Michael. Birds of Passage: Migrant Labor and Industrial Societies Hardcover, 1979.

PORTES Alejandro. "Conclusion: Towards a new World: The origins and effects of transnational activities" Ethnic and Racial Studies Vol. 22, (2): 463-477, 1999.

RONIGER, L. "The Latin-American community of Israel : some notes on Latin-American Jews and Latin American-Israelis". Israel Social Science Research 6 (1): 63-72, 1998-1989.

RONIGER, L. and JAROCHEVSKY, G. "Latin-Americans in Israel: the invisible community". Reflejos. Journal of the Department of Latin-American and Spanish Studies. The Hebrew University of Jerusalem 1 (1): 39-49. (In Spanish), 1992.

SAPIR, E. "The contribution of psychiatry to an understanding of behavior in society" American Journal Sociology (42): 862-870, 1937.

SHEFFER, Gabriel. "Israel in Diaspora Jewish Identity". EN: BEN MOSHE Dianny and SEGEV Zohar (Eds). Israel, Diaspora and Jewish Identity Sussex University Press, 2007.

SHUTZ A. "The Phenomenology of the Social World". George Walsh and Frederick Lehnert, translators. Evanston, Northwestern University Press, 1967.

SHUVAL J. y LESHEM E, "The Sociology of migration in Israel: A critical view" EN: SHUVAL J. and LESHEM E. (Eds). Inmigration to Israel, Sociologial Perspectives New Brunswick: Translation Publishers, 1998.

SOYSAL, Yasemin N. Limits of Citizenship. Migrants and Postnational Membership in Europe The University of Chicago Press, 1994.

TAFT, R. "A psychological Model for the Study of Social Assimilation" Human Relations 10: 141-156, Sage Journals, 1957.

TYLER V. Lynn. Intercultural Integrating David M. Kennedy Center for International Studies, Brigham Young University, 1987. 